\title{
SOME EQUIVALENT FORMULATIONS OF UNIVERSALITY LIMITS IN THE BULK
}

\author{
ELI LEVIN ${ }^{1}$ AND DORON S. LUBINSKY ${ }^{2}$ \\ Dedicated to the 60th Birthday of Bill Lopez
}

\begin{abstract}
We present some equivalences for universality limits in the bulk, involving partial derivatives of reproducing kernels, and spacing of zeros of reproducing kernels.
\end{abstract}

\section{Introduction And Results}

Let $\mu$ be a finite positive Borel measure with compact support $\operatorname{supp}[\mu]$ and infinitely many points in the support. Then we may define orthonormal polynomials

$$
p_{n}(x)=\gamma_{n} x^{n}+\ldots, \gamma_{n}>0,
$$

$n=0,1,2, \ldots$ satisfying the orthonormality conditions

$$
\int p_{n} p_{m} d \mu=\delta_{m n}
$$

Throughout we use

$$
w=\frac{d \mu}{d x}
$$

to denote the Radon-Nikodym derivative of $\mu$.

Orthogonal polynomials play an important role in random matrix theory [3], [7], [29]. One of the key limits there involves the reproducing kernel

$$
K_{n}(x, y)=\sum_{k=0}^{n-1} p_{k}(x) p_{k}(y) .
$$

Because of the Christoffel-Darboux formula, it may also be expressed as

$$
K_{n}(x, y)=\frac{\gamma_{n-1}}{\gamma_{n}} \frac{p_{n}(x) p_{n-1}(y)-p_{n-1}(x) p_{n}(y)}{x-y} .
$$

Define the normalized kernel

$$
\widetilde{K}_{n}(x, y)=w(x)^{1 / 2} w(y)^{1 / 2} K_{n}(x, y) .
$$

Date: November 16, 2008.

1991 Mathematics Subject Classification. AMS(MOS) Classification: 15A52, 42C05.

Key words and phrases. Universality Limits, Random Matrices, Orthogonal Polynomials

Research supported by NSF grant DMS0400446 and US-Israel BSF grant 20004353. 
The simplest case of the universality law is the limit

$$
\lim _{n \rightarrow \infty} \frac{\widetilde{K}_{n}\left(\xi+\frac{a}{\widetilde{K}_{n}(\xi, \xi)}, \xi+\frac{b}{\widetilde{K}_{n}(\xi, \xi)}\right)}{\widetilde{K}_{n}(\xi, \xi)}=\frac{\sin \pi(a-b)}{\pi(a-b)} .
$$

Typically this holds uniformly for $\xi$ in a compact subinterval of $(-1,1)$ and $a, b$ in compact subsets of the real line. Of course, when $a=b$, we interpret $\frac{\sin \pi(a-b)}{\pi(a-b)}$ as 1 .

Perhaps the oldest approach to establishing universality limits has been to substitute asymptotics of sufficiently high order for orthogonal polynomials into the Christoffel-Darboux formula. This was the original approach in the mathematical physics literature. Since the 1990's, Riemann-Hilbert methods have been used to establish high precision asymptotics for orthogonal polynomials, often with a complete asymptotic expansion [2], [3], [10], [11], [12], [26], [27], [28], [45] (this is a very partial list!). This certainly implied universality limits with error terms, amongst many other things. There are also a number of other methods from mathematical physics [4], [6], [9], [32], [33], and from functional and classical analysis [42], [43], [44], [46]. A different setting for universality, where the entries of the random matrices are independently distributed has also been widely studied - see for example [37].

The realization that first order asymptotics, such as

$$
p_{n}(\cos \theta)=\cos n \theta+o(1), n \rightarrow \infty,
$$

are sufficient for universality, was made in [14]. It turns out that when substituting into the Christoffel-Darboux formula, the error term can then be controlled with the aid of a Markov-Bernstein inequality. However, even first order asymptotics for orthogonal polynomials are not necessary for universality - in [19], it was observed that, essentially, all one needs are asymptotics (or even ratio asymptotics) for the Christoffel function

$$
\lambda_{n}(x)=1 / K_{n}(x, x)=1 / \sum_{j=0}^{n-1} p_{j}^{2}(x) .
$$

Christoffel functions have been studied for decades [25], [31], [34], [36], [40], and are far easier to handle than orthogonal polynomials and the reproducing kernel $K_{n}(x, t)$, because they admit the extremal property

$$
\lambda_{n}(x)=\inf _{\operatorname{deg}(P) \leq n-1} \frac{\int P^{2} d \mu}{P^{2}(x)} .
$$

Thus $\lambda_{n}$ is an increasing function of the measure $\mu$.

The main idea of [19] was that one can start with a nice classical weight, such as the Legendre weight, for which universality is known, and can then extend this to much more general measures, using a localization argument. The crux of the extension method is the following inequality: let $\mu^{*}$ be 
another measure with reproducing kernel $K_{n}^{*}(x, y)$ and Christoffel function $\lambda_{n}^{*}(x)$. Assume that $\mu^{*} \geq \mu$. Then for all real $x$ and $y$,

$$
\begin{aligned}
\frac{\left|K_{n}(x, y)-K_{n}^{*}(x, y)\right|}{K_{n}(x, x)} & \leq\left(\frac{K_{n}(y, y)}{K_{n}(x, x)}\right)^{1 / 2}\left(1-\frac{K_{n}^{*}(x, x)}{K_{n}(x, x)}\right)^{1 / 2} \\
& =\left(\frac{\lambda_{n}(x)}{\lambda_{n}(y)}\right)\left(1-\frac{\lambda_{n}(x)}{\lambda_{n}^{*}(x)}\right)^{1 / 2} .
\end{aligned}
$$

Note that on the right-hand side we have only Christoffel functions.

In [19], it was assumed that $\mu$ is a finite positive measure with support $\operatorname{supp}[\mu]=[-1,1]$, that is regular (in the sense of Stahl and Totik [38]), meaning that

$$
\lim _{n \rightarrow \infty} \gamma_{n}^{1 / n}=\frac{1}{\operatorname{cap}(\operatorname{supp}[\mu])},
$$

where cap denotes logarithmic capacity. Moreover, it was assumed that in a neighborhood of some compact set $J \subset(-1,1), \mu$ is absolutely continuous, while $w=\mu^{\prime}$ is positive and continuous at each point of $J$. The universality limit (1.4) was established uniformly for $\xi \in J$ and $a, b$ in compact subsets of the real line. If $J$ consists of just a single point $x$, then the hypothesis is that $\mu$ is absolutely continuous in some neighborhood $(x-\varepsilon, x+\varepsilon)$ of $x$, while $w(x)>0$ and $w$ is continuous at $x$. This alone is sufficient for universality at $x$.

Totik [41], his student Findley [5], and Simon [35] presented far reaching extensions of this result. For example, Totik showed that the same result holds for regular measures on a general compact subset of the real line, instead of $[-1,1]$, and moreover, we may relax the requirement of continuity of $w$. We only need $\log w$ to be integrable in a neighborhood of the points where universality is desired, together with a Lebesgue point type condition on a certain local Szegö function. In particular, we obtain universality a.e. in any neighborhood where $\log w$ is integrable.

Totik's method was based on that in [19], together with "polynomial pullbacks" and potential theory. The former involves sets of the form $P^{[-1]}[-1,1]=\{x: P(x) \in[-1,1]\}$, where $P$ is a polynomial. One of the main technical problems Totik faced was the lack of a suitable weight with given non-classical support for which universality is known. He used these polynomial pullbacks to manufacture one.

Simon [35] used the ideas of [19] together with Jost functions, and other tools, to prove universality at points of continuity for any regular measure. We emphasize that all these results require regularity of the measure $\mu$, and use this property in an essential way. This circle of methods has also been exploited for orthogonal polynomials on the unit circle, and for universality at the edge of the spectrum [15], [16], [20], [21].

A drawback of the comparison method is that applies only to regular measures. To attempt to circumvent this, in [22], another method was introduced, based on the theory of entire functions of exponential type, that 
works for arbitrary, possibly non-regular, measures with compact support. The main result was that universality is equivalent to "universality along the diagonal", or alternatively, ratio asymptotics for Christoffel functions:

\section{Theorem 1.1}

Let $\mu$ be a finite positive Borel measure on the real line with compact support. Let $J \subset \operatorname{supp}[\mu]$ be compact, and such that $\mu$ is absolutely continuous in an open set containing J. Assume that $w$ is positive and continuous at each point of $J$. The following are equivalent:

(I) Uniformly for $\xi \in J$ and a in compact subsets of the real line,

$$
\lim _{n \rightarrow \infty} \frac{K_{n}\left(\xi+\frac{a}{n}, \xi+\frac{a}{n}\right)}{K_{n}(\xi, \xi)}=1 .
$$

(II) Uniformly for $\xi \in J$ and $a, b$ in compact subsets of the complex plane, we have

$$
\lim _{n \rightarrow \infty} \frac{K_{n}\left(\xi+\frac{a}{\widetilde{K}_{n}(\xi, \xi)}, \xi+\frac{b}{\widetilde{K}_{n}(\xi, \xi)}\right)}{K_{n}(\xi, \xi)}=\frac{\sin \pi(a-b)}{\pi(a-b)} .
$$

\section{Remarks}

Instead of assuming continuity on $J$, we can assume a Lebesgue point type condition. It was Vili Totik who first observed that universality can be proved at Lebesgue points, rather than just points of continuity [41].

\section{Theorem 1.2}

Let $\mu$ be a finite positive Borel measure with compact support. Let $J \subset$ $\operatorname{supp}[\mu]$ be compact, and such that $\mu$ is absolutely continuous in an open set containing J. Assume that $w$ is bounded above and below by positive constants in that open set. Assume, moreover, that uniformly for $\xi \in J$, we have

$$
\lim _{s \rightarrow 0+} \frac{1}{s} \int_{\xi-s}^{\xi+s}|w(t)-w(\xi)| d t=0 .
$$

Then the equivalence of (I), (II) in Theorem 1.1 remains valid.

Of course, when $J=\{\xi\}$, (1.7) just asserts that $\xi$ is a Lebesgue point of $w$. When $J$ has non-empty interior, the uniformity of (1.7) forces $w$ to be continuous in the interior of $J$.

The method of [22] has been used in [1], [17], [18], [23], [24]. In this paper, we present a number of other equivalence conditions, summarized in the following theorem. Throughout, we let

$$
K_{n}^{(r, s)}(x, y)=\sum_{k=0}^{n-1} p_{k}^{(r)}(x) p_{k}^{(s)}(y)
$$


and

$$
\tilde{K}_{n}^{(r, s)}(x, y)=w^{1 / 2}(x) w^{1 / 2}(y) K_{n}^{(r, s)}(x, y)
$$

for non-negative integers $r, s$. We also define

$$
\tau_{r, s}=\left\{\begin{array}{rr}
0, & r+s \text { odd } \\
\frac{(-1)^{(r-s) / 2}}{r+s+1}, & r+s \text { even }
\end{array} .\right.
$$

Given a real number $\xi$, we let $\left\{t_{j, n}\right\}_{j}=\left\{t_{j, n}(\xi)\right\}_{j}$ denote the $n-1$ or $n$ zeros of the polynomial

$$
(t-\xi) K_{n}(\xi, t)=\frac{\gamma_{n-1}}{\gamma_{n}}\left(p_{n}(t) p_{n-1}(\xi)-p_{n-1}(t) p_{n}(\xi)\right) .
$$

It is a classical result that they are all real and simple [8, p.19]. Note too that one of these is $\xi$, and if $\xi$ is a zero of $p_{n}$, then $\left\{t_{j n}\right\}_{j}$ are just the zeros of $p_{n}$. We shall assume they are indexed so that $\left\{t_{j n}\right\}$ is increasing. More precisely, we assume

$$
\ldots<t_{-2, n}(\xi)<t_{-1, n}(\xi)<t_{0, n}(\xi)=\xi<t_{1, n}(\xi)<t_{2, n}(\xi)<\ldots
$$

Of course it is possible that all $t_{k, n}$, other than $\xi$, lie to the left or right of $\xi$. Below $\operatorname{dist}(x, J)$ denotes the distance from a point $x$ to a set $J$.

\section{Theorem 1.3}

Assume the hypotheses of Theorem 1.2. The following are equivalent:

(I) or (II) above hold;

(III) For each $r, s \geq 0$, and uniformly for $\xi \in J$,

$$
\lim _{n \rightarrow \infty} \frac{\tilde{K}_{n}^{(r, s)}(\xi, \xi)}{\widetilde{K}_{n}(\xi, \xi)^{r+s+1}}=\pi^{r+s} \tau_{r, s} .
$$

(IV) There exists $L>0$, such that uniformly in $n$ and for $\xi$ with dist $(\xi, J) \leq$ $L / n$,

$$
K_{n}^{(1,0)}(\xi, \xi)=o\left(n^{2}\right) .
$$

If we assume also $w$ is continuous in $J$, there is the additional equivalence: $(V)$ For each fixed $j$, we have uniformly in $\xi \in J$,

$$
\lim _{n \rightarrow \infty}\left(t_{n, j+1}(\xi)-t_{n, j}(\xi)\right) \tilde{K}_{n}(\xi, \xi)=1 .
$$

Thus, universality is equivalent to asymptotics for derivatives of the reproducing kernels, or just weak growth estimates on $K_{n}^{(1,0)}$. Moreover, when $w$ is continuous, universality is equivalent to "clock spacing" of zeros of the reproducing kernel, in the terminology of Barry Simon. See the papers [13], [36], [41] for more details on the relation between universality and varying assumptions on zero spacing. We prove Theorem 1.3 in Section 2. 


\section{Proofs}

In the sequel $C, C_{1}, C_{2}, \ldots$ denote constants independent of $n, x, y, s, t$. The same symbol does not necessarily denote the same constant in different occurences. We shall write $C=C(\alpha)$ or $C \neq C(\alpha)$ to respectively denote dependence on, or independence of, the parameter $\alpha$. We use $\sim$ in the following sense: given real sequences $\left\{c_{n}\right\},\left\{d_{n}\right\}$, we write

$$
c_{n} \sim d_{n}
$$

if there exist positive constants $C_{1}, C_{2}$ with

$$
C_{1} \leq c_{n} / d_{n} \leq C_{2} \text {. }
$$

Similar notation is used for functions and sequences of functions.

Let $\left\{\xi_{n}\right\}$ denote a sequence in $J$, and for $n \geq 1$,

$$
f_{n}(a, b)=\frac{K_{n}\left(\xi_{n}+\frac{a}{\widetilde{K}_{n}\left(\xi_{n}, \xi_{n}\right)}, \xi_{n}+\frac{b}{\widetilde{K}_{n}\left(\xi_{n}, \xi_{n}\right)}\right)}{K_{n}\left(\xi_{n}, \xi_{n}\right)} .
$$

As noted above, the equivalence of (I) and (II) is the main result of [22]. We shall prove $(\mathrm{II}) \Leftrightarrow(\mathrm{III}) ;(\mathrm{I}) \Longleftrightarrow(\mathrm{IV})$ and $(\mathrm{II}) \Longleftrightarrow(\mathrm{V})$. We begin by summarizing some results from [22]. Recall too that the exponential type $A$ of an entire function $g$ is

$$
A=\limsup _{r \rightarrow \infty} \frac{\log \left(\max _{|z|=r}|g(z)|\right)}{r},
$$

if this number is finite.

\section{Lemma 2.1}

(a) $\left\{f_{n}\right\}_{n=1}^{\infty}$ is uniformly bounded in compact subsets of $\mathbb{C}$. Let $f(a, b)$ be the limit of some subsequence $\left\{f_{n}(\cdot, \cdot)\right\}_{n \in \mathcal{S}}$ of $\left\{f_{n}(\cdot, \cdot)\right\}_{n=1}^{\infty}$. It is an entire function of exponential type in $a, b$, that satisfies for all complex $a, b$,

$$
|f(a, b)| \leq C_{1} e^{C_{2}(|\operatorname{Im} a|+|\operatorname{Im} b|)} .
$$

(b) For all $a \in \mathbb{C}$,

$$
\int_{-\infty}^{\infty}|f(a, s)|^{2} d s \leq f(a, \bar{a})
$$

(c) Let $\sigma$ be the exponential type of $f(a, \cdot)$. This is independent of $a \in \mathbb{R}$, and

$$
\sigma=\pi \sup _{x \in \mathbb{R}} f(x, x) .
$$

(d) For real a, the function $f(a, \cdot)$ has only real zeros.

\section{Proof}

(a) This is Lemma 5.2(a) and (b) in [22].

(b) This is Lemma 5.3(b) in [22].

(c) This is Lemma 6.1 and 6.4 in [22].

(d) This is Lemma 5.2(c) in [22]. 
Proof of (II) $\Rightarrow$ (III)

This is similar to Corollary 1.3 in [19], and generalizes that corollary. Expanding $f_{n}$ as a double Taylor series gives

$$
\begin{aligned}
& f_{n}(a, b) \\
& =\sum_{r, s=0}^{\infty} \frac{K_{n}^{(r, s)}\left(\xi_{n}, \xi_{n}\right)}{r ! s ! K_{n}\left(\xi_{n}, \xi_{n}\right)}\left(\frac{a}{\widetilde{K}_{n}\left(\xi_{n}, \xi_{n}\right)}\right)^{r}\left(\frac{b}{\widetilde{K}_{n}\left(\xi_{n}, \xi_{n}\right)}\right)^{s} .
\end{aligned}
$$

By using the Maclaurin series of sin and the binomial theorem, we see that

$$
\frac{\sin \pi(a-b)}{\pi(a-b)}=\sum_{r, s=0}^{\infty} \frac{(a \pi)^{r}(b \pi)^{s}}{r ! s !} \tau_{r, s} .
$$

Thus

$$
\begin{aligned}
& f_{n}(a, b)-\frac{\sin \pi(a-b)}{\pi(a-b)} \\
= & \sum_{r, s=0}^{\infty} \frac{a^{r} b^{s}}{r ! s !}\left\{\frac{K_{n}^{(r, s)}\left(\xi_{n}, \xi_{n}\right)}{\widetilde{K}_{n}\left(\xi_{n}, \xi_{n}\right)^{r+s} K_{n}\left(\xi_{n}, \xi_{n}\right)}-\pi^{r+s} \tau_{r, s}\right\} .
\end{aligned}
$$

Since our hypothesis is that $f_{n}(a, b)-\frac{\sin \pi(a-b)}{\pi(a-b)}$ converges uniformly to 0 for $a, b$ in compact subsets of the plane, we deduce that for each fixed $r, s \geq 0$,

$$
\lim _{n \rightarrow \infty} \frac{K_{n}^{(r, s)}\left(\xi_{n}, \xi_{n}\right)}{\widetilde{K}_{n}\left(\xi_{n}, \xi_{n}\right)^{r+s} K_{n}\left(\xi_{n}, \xi_{n}\right)}=\pi^{r+s} \tau_{r, s} .
$$

Since $\left\{\xi_{n}\right\}$ is any sequence in $J$, we have shown that uniformly for $\xi \in J$,

$$
\lim _{n \rightarrow \infty} \frac{K_{n}^{(r, s)}(\xi, \xi)}{\widetilde{K}_{n}(\xi, \xi)^{r+s} K_{n}(\xi, \xi)}=\lim _{n \rightarrow \infty} \frac{\tilde{K}_{n}^{(r, s)}(\xi, \xi)}{\widetilde{K}_{n}(\xi, \xi)^{r+s+1}}=\pi^{r+s} \tau_{r, s} .
$$

Proof of $($ III $) \Rightarrow($ II $)$

We essentially retrack the steps of the proof of (II) $\Rightarrow$ (III). By Lemma 2.1(a), $\left\{f_{n}(a, b)-\frac{\sin \pi(a-b)}{\pi(a-b)}\right\}_{n>1}$ is a sequence of analytic functions that is uniformly bounded in compact subsets of the plane. Moreover our hypothesis is that individual Maclaurin series coefficients in the double series converge to 0 as $n \rightarrow \infty$. Classical complex analysis then shows that

$$
\lim _{n \rightarrow \infty}\left(f_{n}(a, b)-\frac{\sin \pi(a-b)}{\pi(a-b)}\right)=0
$$

uniformly in compact subsets of the plane. Since $\left\{\xi_{n}\right\}$ in the definition of $f_{n}$ is any sequence in $J$, we obtain the stated uniformity in $\xi$ in (1.6).

We note that (III) does not immediately imply (IV) because the latter involves points that lie outside $J$. 
Proof of $(\mathrm{I}) \Rightarrow(\mathrm{IV})$

By hypothesis,

$$
\lim _{n \rightarrow \infty} f_{n}(a, a)=1
$$

uniformly for $a$ in compact subsets of the plane. Then also

$$
\lim _{n \rightarrow \infty} \frac{d}{d a} f_{n}(a, a)=0,
$$

uniformly for $a$ in compact subsets of the plane, that is uniformly in such $a$,

$$
\lim _{n \rightarrow \infty} \frac{2 K_{n}^{(1,0)}\left(\xi_{n}+\frac{a}{\widetilde{K}_{n}\left(\xi_{n}, \xi_{n}\right)}, \xi_{n}+\frac{a}{\widetilde{K}_{n}\left(\xi_{n}, \xi_{n}\right)}\right)}{K_{n}\left(\xi_{n}, \xi_{n}\right) \widetilde{K}_{n}(\xi, \xi)}=0 .
$$

Since we assumed that $w=\mu^{\prime} \sim 1$ uniformly in a neighborhood of $J$, a standard estimate for Christoffel functions [30, Theorem 20, p. 116] gives

$$
\widetilde{K}_{n}(\xi, \xi) \sim K_{n}(\xi, \xi) \sim n .
$$

The result then follows.

Proof of $(\mathrm{IV}) \Rightarrow(\mathrm{I})$

Let $|a| \leq L$. By the Mean Value Theorem, for some $t$ between $\xi$ and $\xi+\frac{a}{n}$,

$$
\begin{aligned}
& \frac{K_{n}\left(\xi+\frac{a}{n}, \xi+\frac{a}{n}\right)}{K_{n}(\xi, \xi)}-1 \\
= & \frac{1}{K_{n}(\xi, \xi)} \frac{a}{n} \frac{\partial}{\partial t}\left(K_{n}(t, t)\right) \\
= & \frac{1}{K_{n}(\xi, \xi)} \frac{a}{n} 2 K_{n}^{(1,0)}(t, t)=o(1),
\end{aligned}
$$

uniformly in $\xi \in J$ by our hypothesis and (2.5). It then follows that for some $R>0$, we have

$$
\lim _{n \rightarrow \infty} f_{n}(a, a)=1
$$

uniformly for $|a| \leq R$. In view of the uniform boundedness of $\left\{f_{n}\right\}$, convergence continuation theorems gives this for all real (and even complex) $a$. Since $\left\{\xi_{n}\right\}$ in the definition of $\left\{f_{n}\right\}$ is any sequence in $J,(1.5)$ follows uniformly for $\xi \in J$.

The most difficult equivalence concerns the spacing of the zeros:

\section{Proof of $(\mathrm{II}) \Rightarrow(\mathrm{V})$}

It is now a well established fact, first observed by the first author in [15], that the universality (1.7) implies "clock spacing" of zeros. The latter is a phrase coined by Barry Simon. It has been analyzed in a number of contexts, and in weaker and stronger forms, by Last, Simon and others (see [13], [36]). Although established in [15] only for the zeros of the orthogonal polynomials (that is the special case where $\xi$ is a zero of $p_{n}$ ), the exact same proof works for the case stated here. The main idea is that the uniform 
convergence in (1.6) and Hurwitz's theorem imply that as $n \rightarrow \infty$, the zeros of $K_{n}\left(\xi_{n}, \xi_{n}+\frac{z}{\widetilde{K}_{n}\left(\xi_{n}, \xi_{n}\right)}\right)$ converge to those of $\frac{\sin \pi z}{\pi z}$. Because $\left\{\xi_{n}\right\}$ is any sequence in $J$, we obtain the stated uniformity in $\xi$.

To prove $(\mathrm{V}) \Rightarrow(\mathrm{I})$, we shall need:

\section{Lemma 2.2}

Assume the hypotheses of Theorem 1.2. Assume also that if $\left\{\xi_{n}\right\}$ is a sequence in $J$, then for each fixed $j$,

$$
\lim _{n \rightarrow \infty}\left(\frac{t_{n, j+1}\left(\xi_{n}\right)-t_{n, j}\left(\xi_{n}\right)}{t_{n, 1}\left(\xi_{n}\right)-t_{n, 0}\left(\xi_{n}\right)}\right)=1 .
$$

Let $f$ be as in the previous lemma. Then

$$
f(z, w)=\sum_{k=-\infty}^{\infty} f\left(k \frac{\pi}{\sigma}, k \frac{\pi}{\sigma}\right) \frac{\sin (\sigma z-k \pi)}{\sigma z-k \pi} \frac{\sin (\sigma w-k \pi)}{\sigma w-k \pi} .
$$

Moreover, if for each $k \in \mathbb{Z}$,

$$
f\left(k \frac{\pi}{\sigma}, k \frac{\pi}{\sigma}\right)=1
$$

then

$$
f(z, w)=\frac{\sin \pi(z-w)}{\pi(z-w)} .
$$

\section{Remark}

Barry Simon calls the limit (2.6) "weak clock" behavior.

Proof

Let $\left\{\rho_{j}\right\}_{j \neq 0}$ denote the zeros of $f(0, z)$ in increasing order, and let $\rho_{0}=0$. By Hurwitz' Theorem,

$$
\rho_{j}=\lim _{n \rightarrow \infty, n \in \mathcal{S}} \rho_{j, n}
$$

where $\left\{\rho_{j, n}\right\}$ are the zeros of $f_{n}(0, z)$, appropriately ordered. Note that with an appropriate ordering,

$$
\rho_{j, n}=K_{n}\left(\xi_{n}, \xi_{n}\right)\left(t_{j, n}-\xi_{n}\right) .
$$

Then our spacing assumption (2.6) gives, perhaps with a reindexation of the zeros,

$$
\frac{\rho_{j+1}-\rho_{j}}{\rho_{1}-\rho_{0}}=\lim _{n \rightarrow \infty, n \in \mathcal{S}}\left(\frac{t_{n, j+1}\left(\xi_{n}\right)-t_{n, j}\left(\xi_{n}\right)}{t_{n, 1}\left(\xi_{n}\right)-t_{n, 0}\left(\xi_{n}\right)}\right)=1 .
$$

Thus setting $\Delta=\rho_{1}$, and recalling $\rho_{0}=0$, we have

$$
\rho_{j}=j \Delta, j \in \mathbb{Z} \text {. }
$$

Note too that if $j \neq \ell$, then

$$
f_{n}\left(\rho_{j, n}, \rho_{\ell, n}\right)=\frac{K_{n}\left(t_{j, n}, t_{\ell, n}\right)}{K_{n}\left(\xi_{n}, \xi_{n}\right)}=0
$$


so for $j \neq \ell$,

$$
f(j \Delta, \ell \Delta)=f\left(\rho_{j}, \rho_{\ell}\right)=0 .
$$

The spacing assumption (2.6) ensures that all zeros $\left\{\rho_{j}\right\}_{j \neq 0}=\{j \Delta\}_{j \neq 0}$ are simple zeros of $f(0, z)$. Next, let

$$
g(z)=f(0, z) /\left[\frac{\sin \frac{\pi}{\Delta} z}{\frac{\pi}{\Delta} z}\right]
$$

This is entire, and has no zeros, and satisfies $g(0)=1$. Moreover, it is a ratio of entire functions of exponential type, so has exponential type. By the Hadamard factorization theorem, it must have the form

$$
g(z)=e^{C z}
$$

for some constant $C$. Since $g$ is real valued on the real line, $C$ must be real. But then for all $j \in \mathbb{Z}$,

$$
\left|f\left(0, \Delta\left(j+\frac{1}{2}\right)\right)\right|=\frac{e^{C \Delta\left(j+\frac{1}{2}\right)}}{\pi\left|j+\frac{1}{2}\right|} .
$$

This contradicts the fact that $f(0, \cdot)$ is bounded on the real axis unless $C=0$. Thus

$$
f(0, z)=\frac{\sin \frac{\pi}{\Delta} z}{\frac{\pi}{\Delta} z}
$$

and in particular, the exponential type of $f(0, \cdot)$, which we called $\sigma$, equals $\frac{\pi}{\Delta}$. By Lemma 2.1(c), for any real $a, f(a, \cdot)$ then has exponential type $\frac{\pi}{\Delta}$. Since also $f(a, \cdot) \in L_{2}(\mathbb{R})$, (recall Lemma 2.1(b)), we can apply the cardinal series expansion [39, p. 91]

$$
f(a, z)=\sum_{k=-\infty}^{\infty} f(a, k \Delta) \frac{\sin \left(\frac{\pi}{\Delta} z-k \pi\right)}{\frac{\pi}{\Delta} z-k \pi} .
$$

In turn, $f(\cdot, k \Delta)$ is an entire function of exponential type $\frac{\pi}{\Delta}$ that belongs to $L_{2}(\mathbb{R})$, so applying the cardinal series expansion again, gives

$$
f(a, z)=\sum_{k=-\infty}^{\infty}\left[\sum_{j=-\infty}^{\infty} f(j \Delta, k \Delta) \frac{\sin \left(\frac{\pi}{\Delta} a-j \pi\right)}{\frac{\pi}{\Delta} a-j \pi}\right] \frac{\sin \left(\frac{\pi}{\Delta} z-k \pi\right)}{\frac{\pi}{\Delta} z-k \pi} .
$$

In view of (2.10), we obtain for all real $a$, and all complex $z$,

$$
f(a, z)=\sum_{k=-\infty}^{\infty} f(k \Delta, k \Delta) \frac{\sin \left(\frac{\pi}{\Delta} a-k \pi\right)}{\frac{\pi}{\Delta} a-k \pi} \frac{\sin \left(\frac{\pi}{\Delta} z-k \pi\right)}{\frac{\pi}{\Delta} z-k \pi} .
$$

By analytic continuation, this extends to all complex $a$ as well. Recalling that $\frac{\pi}{\Delta}=\sigma$, we obtain $(2.7)$. 
Finally if (2.8) holds, then

$$
f(a, z)=\sum_{k=-\infty}^{\infty} \frac{\sin (\sigma a-k \pi)}{\sigma a-k \pi} \frac{\sin (\sigma z-k \pi)}{\sigma z-k \pi}=\frac{\sin \sigma(a-z)}{\sigma(a-z)}
$$

by applying this identity to the special function $\frac{\sin \sigma(a-z)}{\sigma(a-z)}$. In particular, then for all real $x$,

$$
f(x, x)=1,
$$

so by (2.5), $\sigma=\pi \sup _{x \in \mathbb{R}} f(x, x)=\pi$ and (2.9) also follows.

\section{Proof of $(\mathrm{V}) \Rightarrow(\mathrm{II})$}

Our hypothesis (1.12) implies the weak clock spacing (2.6) of Lemma 2.2. The result then follows if we can show that for all $k, f\left(k \frac{\pi}{\sigma}, k \frac{\pi}{\sigma}\right)=1$. Our hypothesis shows that for each sequence $\left\{\xi_{n}\right\}$ in $J$, and each fixed $j$,

$$
\lim _{n \rightarrow \infty}\left(t_{n, j+1}\left(\xi_{n}\right)-t_{n, j}\left(\xi_{n}\right)\right) \tilde{K}_{n}\left(\xi_{n}, \xi_{n}\right)=1 .
$$

Fix an integer $\ell$, and set

$$
\xi_{n}^{\prime}=t_{n, \ell}\left(\xi_{n}\right) .
$$

It then follows that, as sets,

$$
\left\{t_{j n}\left(\xi_{n}^{\prime}\right)\right\}=\left\{t_{j n}\left(\xi_{n}\right)\right\} .
$$

Our hypothesis (1.12) gives

$$
\lim _{n \rightarrow \infty}\left(t_{n, 1}\left(\xi_{n}^{\prime}\right)-t_{n, 0}\left(\xi_{n}^{\prime}\right)\right) \tilde{K}_{n}\left(\xi_{n}^{\prime}, \xi_{n}^{\prime}\right)=1,
$$

or equivalently

$$
\lim _{n \rightarrow \infty}\left(t_{n, \ell+1}\left(\xi_{n}\right)-t_{n, \ell}\left(\xi_{n}\right)\right) \tilde{K}_{n}\left(\xi_{n}^{\prime}, \xi_{n}^{\prime}\right)=1 .
$$

Together, this and (2.11) give

$$
\lim _{n \rightarrow \infty} \frac{\tilde{K}_{n}\left(t_{n, \ell}\left(\xi_{n}\right), t_{n, \ell}\left(\xi_{n}\right)\right)}{\tilde{K}_{n}\left(\xi_{n}, \xi_{n}\right)}=\lim _{n \rightarrow \infty} \frac{\tilde{K}_{n}\left(\xi_{n}^{\prime}, \xi_{n}^{\prime}\right)}{\tilde{K}_{n}\left(\xi_{n}, \xi_{n}\right)}=1,
$$

or equivalently

$$
\lim _{n \rightarrow \infty} \frac{w\left(t_{n, \ell}\left(\xi_{n}\right)\right)}{w\left(\xi_{n}\right)} f_{n}\left(\rho_{\ell, n}, \rho_{\ell, n}\right)=1
$$

and hence for each $\ell$,

$$
f\left(\rho_{\ell}, \rho_{\ell}\right)=1 \text {. }
$$

Since in our earlier notation, $\rho_{j}=j \Delta=j \frac{\pi}{\sigma}$, we have (2.8). As the limit function $f(z, w)=\frac{\sin \pi(z-w)}{\pi(z-w)}$ is independent of the subsequence $\left\{f_{n}\right\}_{n \in \mathcal{S}}$ from which $f$ was formed, the result now follows from the previous lemma. 


\section{REFERENCES}

[1] A. Avila, J. Last, and B. Simon, Bulk universality and clock spacing of zeros for ergodic Jacobi matrices with a.c. spectrum, submitted

[2] J. Baik, T. Kriecherbauer, K. T-R. McLaughlin, P.D. Miller, Uniform Asymptotics for Polynomials Orthogonal with respect to a General Class of Discrete Weights and Universality Results for Associated Ensembles, Princeton Annals of Mathematics Studies, 2006.

[3] P. Deift, Orthogonal Polynomials and Random Matrices: A Riemann-Hilbert Approach, Courant Institute Lecture Notes, Vol. 3, New York University Pres, New York, 1999.

[4] P. Desrosiers, P.J. Forrester, Hermite and Laguerre $\beta$-ensembles: asymptotic corrections to the eigenvalue density, Nuclear Phys. B 743 (2006), no. 3, 307-332.

[5] M. Findley, Universality for Regular Measures satisfying Szegö's Condition, to appear in J. Approx. Theory.

[6] P. J. Forrester, Spacing distributions in random matrix ensembles, (in) Recent perspectives in random matrix theory and number theory, London Math. Soc. Lecture Note Ser., 322, Cambridge Univ. Press, Cambridge, 2005, pp. 279-307.

[7] P. J. Forrester, Log-gases and Random matrices, online book, http://www.ms.unimelb.edu.au/ matpjf/matpjf.html .

[8] G. Freud, Orthogonal Polynomials, Pergamon Press/ Akademiai Kiado, Budapest, 1971.

[9] T.M. Garoni, P.J. Forrester, N. E. Frankel, Asymptotic corrections to the eigenvalue density of the GUE and LUE, J. Math. Phys., 46 (2005), no. 10, 103301, 17 pp.

[10] A.R. Its, A.B. Kuijlaars, and J. Ostensson, Critical edge behavior in unitary random matrix ensembles and the thirty fourth Painlevé transcendent, International Maths. Research Notices, 2008, article ID rnn017, 67 pages.

[11] A.B. Kuijlaars and M. Vanlessen, Universality for Eigenvalue Correlations from the Modified Jacobi Unitary Ensemble, International Maths. Research Notices, 30(2002), 1575-1600.

[12] A.B. Kuijlaars and M. Vanlessen, Universality for eigenvalue correlations at the origin of the spectrum, Comm. Math. Phys. 243 (2003), 163-191.

[13] Y. Last and B. Simon, Fine Structure of the Zeros of Orthogonal Polynomials IV. A Priori Bounds and Clock Behavior, Comm. Pure Appl. Math. 61 (2008), 486-538.

[14] Eli Levin and D.S. Lubinsky, Universality Limits for Exponential Weights, to appear in Constructive Approximation.

[15] Eli Levin and D.S. Lubinsky, Applications of Universality Limits to Zeros and Reproducing Kernels of Orthogonal Polynomials, Journal of Approximation Theory, 150(2008), 69-95.

[16] Eli Levin and D.S. Lubinsky, Universality Limits Involving Orthogonal Polynomials on the Unit Circle, Computational Methods and Function Theory, 7(2007), 543-561.

[17] Eli Levin and D.S. Lubinsky, Universality Limits in the Bulk for Varying Measures, Advances in Mathematics, 219(2008), 743-779.

[18] Eli Levin and D.S. Lubinsky, Universality Limits at the Soft Edge of the Spectrum via Classical Complex Analysis, manuscript.

[19] D.S. Lubinsky, A New Approach to Universality Limits involving Orthogonal Polynomials, to appear in Annals of Mathematics.

[20] D.S. Lubinsky, A New Approach to Universality Limits at the Edge of the Spectrum, Contemporary Mathematics (60th Birthday of Percy Deift), 458(2008), 281-290.

[21] D.S. Lubinsky, Mutually Regular Measures have Similar Universality Limits, (in) Proceedings of Twelfth Texas Conference on Approximation Theory (eds. M. Neamtu, L. Schumaker), Nashboro Press, Nashville 2008, pp. 256-269. 
[22] D.S. Lubinsky, Universality Limits in the Bulk for Arbitrary Measures with Compact Support, J. d' Analyse de Mathematique, 106(2008), 373-394.

[23] D.S. Lubinsky, Universality Limits at the Hard Edge of the Spectrum for Measures with Compact Support, International Mathematics Research Notices, (2008) 2008: rnn099-39.

[24] D.S. Lubinsky, Universality Limits for Random Matrices and de Branges Spaces of Entire Functions, manuscript.

[25] A. Mate, P. Nevai, V. Totik, Szego's Extremum Problem on the Unit Circle, Annals of Math., 134(1991), 433-453.

[26] K. T.-R. McLaughlin, Asymptotic analysis of random matrices with external source and a family of algebraic curves, Nonlinearity 20 (2007), 1547-1571.

[27] K. T.-R. McLaughlin and P. Miller, The $\bar{\partial}$ steepest descent method and the asymptotic behavior of polynomials orthogonal on the unit circle with fixed and exponentially varying nonanalytic weights, Intern. Math. Res. Papers, 2006, Article ID 48673, pages $1-78$.

[28] K. T.-R. McLaughlin and P. Miller, The $\bar{\partial}$ steepest descent method for orthogonal polynomials on the real line with varying weights, Intern. Math. Res. Notices, 2008, Article ID rnn075, pages 1-66.

[29] M.L. Mehta, Random Matrices, 2nd edn., Academic Press, Boston, 1991.

[30] P. Nevai, Orthogonal Polynomials, Memoirs of the AMS no. 213 (1979).

[31] P. Nevai, Geza Freud, Orthogonal Polynomials and Christoffel Functions: A Case Study, J. Approx. Theory, 48(1986), 3-167.

[32] L. Pastur, From random matrices to quasi-periodic Jacobi matrices via orthogonal polynomials, J. Approx. Theory 139 (2006), 269-292.

[33] L. Pastur and M. Shcherbina, Universality of the local eigenvalue statistics for a class of Unitary Invariant Random Matrix Ensembles, J. Statistical Physics, 86(1997), 109147.

[34] B. Simon, Orthogonal Polynomials on the Unit Circle, Parts 1 and 2, American Mathematical Society, Providence, 2005.

[35] B. Simon, Two Extensions of Lubinsky's Universality Theorem, to appear in Journal d'Analyse de Mathematique.

[36] B. Simon, The Christoffel-Darboux Kernel, to appear in Perspectives in PDE, Harmonic Analysis and Applications, Proceedings of Symposia in Pure and Applied Mathematics.

[37] A. Soshnikov, Universality at the Edge of the Spectrum in Wigner Random Matrices, Comm. Math. Phys., 207(1999), 697-733.

[38] H. Stahl and V. Totik, General Orthogonal Polynomials, Cambridge University Press, Cambridge, 1992.

[39] F. Stenger, Numerical Methods Based on Sinc and Analytic Functions, Springer, New York, 1993.

[40] V. Totik, Asymptotics for Christoffel Functions for General Measures on the Real Line, J. d'Analyse Math., 81(2000), 283-303.

[41] V. Totik, Universality and fine zero spacing on general sets, to appear in Arkiv for Matematik.

[42] C. Tracy, H.L. Widom, Universality of the distribution functions of random matrix theory, (in) Statistical physics on the eve of the 21st century, Ser. Adv. Statist. Mech., 14, World Sci. Publ., River Edge, NJ, 1999, pp. 230-239.

[43] C. Tracy, H.L. Widom, Universality of the distribution functions of random matrix theory, (in) Integrable systems: from classical to quantum (Montréal, QC, 1999), CRM Proc. Lecture Notes, 26, Amer. Math. Soc., Providence, RI, 2000, pp.251-264.

[44] C. Tracy, H.L. Widom, The Pearcey process, Communications in Mathematical Physics, 263 (2006), no. 2, 381-400. 
[45] M. Vanlessen, Strong Asymptotics of Lageurre-type Orthogonal Polynomials and Applications in Random Matrix Theory, Constr. Approx., 25(2007), 125-175.

[46] H. Widom, Toeplitz determinants, random matrices and random permutations, (in) Toeplitz matrices and singular integral equations (Pobershau, 2001), Birkhäuser, Basel, 2002, pp.317-328,

${ }^{1}$ Mathematics Department, The Open University of Israel, P.O. Box 808, RaAnana 43107, Israel., ${ }^{2}$ School of Mathematics, Georgia Institute of Technology, Atlanta, GA 30332-0160, USA., ${ }^{1}$ Lubinsky@Math.Gatech.edu, ${ }^{2}$ Elile@openu.AC.IL 